\title{
A Possible Explanation of Countergradient Fluxes in Homogeneous Turbulence
}

\author{
Thomas Gerz and Ulrich Schumann \\ Institut für Physik der Atmosphäre, DLR Oberpfaffenhofen, \\ D-82230 Weßling, Germany \\ Communicated by Jackson R. Herring
}

Received 26 May 1994 and accepted 7 March 1995

\begin{abstract}
This study addresses the phenomenon of persistent countergradient (PCG) fluxes of momentum and heat (density) as observed in homogeneous turbulence forced by shear and stratification. Countergradient fluxes may occur at large scales when stratification is strong. However, they always occur at small scales, independently of stratification. A conceptional model is introduced to explain PCG fluxes at small scales as the result of the collision of large-scale fluid parcels. The large parcels collide under the driving force of inclined vortex structures (in a shear-dominated flow) or of buoyancy (in a strongly stratified shear flow). This "collision model" also explains the PCG heat flux in an unsheared stratified flow with zero average momentum flux. It is found that the energy of the small-scale PCG motions is provided (i) by quick transport of kinetic energy from the scales of production to relatively slowly dissipating scales if the flow is shear-driven and (ii) by conversion of available potential energy to kinetic energy at small scales when the flow is stratified. The collision mechanism is an inherent property of the turbulence dynamics. Therefore, the PCG fluxes at small scales reflect a universal character of homogeneous turbulence, and are found over a large range of Reynolds numbers. The Prandtl (or Schmidt) number influences the rate of dissipation of temperature (or density) variance but not the dissipation rate of the velocity variance. In stratified flows, therefore, the number directly affects the strength of the PCG heat flux at small scales. It is found, however, that the PCG momentum flux is also altered slightly when the Prandtl number is large enough to sustain small buoyantly moving parcels after collision.
\end{abstract}

\section{Observation}

In a turbulent flow, mean gradients of momentum, density, heat, species concentrations, etc., usually drive respective mean fluxes of sign opposite to the sign of the gradients. These fluxes are called "down-gradient" (DG). Fluid density $\rho$ in air and in water for sufficiently large temperature decreases about linearly with temperature and increases with the concentration of a relatively heavy species like salt in water. Hence, a positive mean vertical temperature gradient or a negative mean vertical salinity gradient cause stable stratification under gravity $g$. Given a stratified flow with, e.g., a positive gradient $s$ of mean temperature $\Theta$ in the vertical direction $z$,

$$
s=\frac{d \Theta}{d z}>0,
$$

the down-gradient turbulent vertical heat flux is negative. In a stratified flow, gravity oscillations may develop which are accompanied by oscillating heat fluxes at a frequency of about $2 N$, where 
$N=(\beta g(d \Theta / d z))^{1 / 2}$ is the Brunt-Väisälä frequency and $\beta$ is the constant volumetric expansion coefficient. Hence, we expect that positive or "countergradient" (CG) heat fluxes occur temporarily, when available potential energy is transferred into kinetic energy of the flow.

However, data obtained from numerical simulations of homogeneous stratified turbulence not only show oscillating fluxes, but often exhibit "persistent countergradient" (PCG) fluxes, i.e., positive fluxes which persist for longer than half the buoyancy period $\pi / N$. Such fluxes develop at small scales and may dominate all scales at strong stratification. The PCG flux phenomena have been reported for heat by Gerz et al. (1989), Gerz and Schumann (1991), Holt et al. (1992), and Ramsden and Holloway (1992). Data from laboratory measurements as well as atmospheric and oceanic field measurements corroborate a similar heat-flux (or density-flux) behavior or, at least, give some evidence for the existence of PCG heat fluxes (Munk, 1981; Komori et al., 1983; Itsweire et al., 1986; Itsweire and Helland, 1989; Lienhard and van Atta, 1990; Yoon and Warhaft, 1990; Salathé and Smith, 1992; Komori and Nagata, 1994).

PCG fluxes contradict general expectations and, therefore, they require special explanation. There is some controversy about PCG fluxes in recent studies, whether it is a large-scale or a small-scale phenomenon, and whether it occurs only in flows with high Prandtl or Schmidt number (as for salt diffusion or thermal diffusion in water) and at moderate Reynolds numbers, or also for thermal diffusion in air at high Reynolds numbers. Both energetic (Schumann, 1987; Holloway, 1988; Gerz et al., 1989; Gerz and Schumann, 1991) and mechanistic (Kaltenbach et al., Holt et al., 1992) explanations have been proposed. In this note we point out that CG fluxes occur at large and at small scales, but under different conditions and flow situations. We illuminate the underlying physics for both phenomena.

Rohr et al. (1988) studied stably stratified homogeneously turbulent shear flows experimentally in a salt-stratified water channel with a turbulent Reynolds number varying between 100 and 200 (based on root-mean square velocity and microlength scale) and a molecular Schmidt number of about 700 . Kaltenbach et al. (1994) performed large-eddy and direct numerical simulations of the same kind of turbulence but with lower effective turbulence Reynolds numbers between 30 and 70 and with an effective (subgrid-scale) Prandtl number of $P r=1$. In both studies data have been recorded for a variety of gradient Richardson numbers:

$$
R i=\frac{N^{2}}{S^{2}}, \quad \text { where } \quad S=\frac{d U}{d z}
$$

is the shear rate of the mean velocity $U$ in the $x$-direction varying in the vertical direction $z$.

We present and discuss cospectra of vertical fluxes of horizontal momentum, $u w$, and heat, $\theta w$ (or density $\rho w$ ) using data from Rohr's measurements (Rohr et al., 1988) and data from Kaltenbach's LESs, which, in parts, are published in Kaltenbach et al. (1994). We refer the reader to these two publications for details of the measurement and simulation techniques. We denote $u, w, \rho$, and $\theta$ as the fluctuating (turbulent) part of downstream and vertical velocity, density, and temperature, respectively. We note that a density flux produces the same buoyancy flux as a heat flux of opposite sign, $\rho w \sim-\beta \rho_{0} \theta w$, where $\rho_{0}$ is the mean density. All quantities are normalized by proper reference scales.

Figure 1 plots measured "downstream" cospectra of $-u w$ and $\rho w$ in variance-preserving form at various downstream positions and for various Richardson numbers (from Figure 20 and 22 of Rohr et al. (1988)). These spectra are obtained by Fourier transforms of temporal fluctuations. By means of Taylor's frozen turbulence hypothesis, they can be interpreted as spectra versus the downstream wave number $k_{x}$ and are called "downstream" spectra, therefore. Figure 2 shows cospectra of $u w$ and $\theta w$ obtained by Fourier transforms of simulated spatial fluctuations in downstream $\left(k_{x}\right)$ and vertical $\left(k_{z}\right)$ directions for $R i=0$ and 0.13 at shear times $S t=6,7$, and 8 . Figure 3 depicts the same quantities for $R i=0.5$ and 1 at $S t=8,9$, and 10 . From the simulations a one-dimensional cospectrum is obtained by averaging the three-dimensional cospectrum over all wave numbers of the other two directions. Rohr et al. emphasize that, owing to limited averaging time, their cospectra of momentum and density fluxes should be interpreted only qualitatively. However and despite and different Reynolds and Prandtl numbers in the measured and simulated flows, the cospectra depicted in Figures 1-3 show the same basic features, and, therefore, demonstrate the same physical states of sheared and stratified turbulence.

Both datasets reveal strong DG fluxes of momentum and heat (density) at large scales for small values of the Richardson number $(R i \leq 0.13)$, which is consistent with a flow state of turbulent mixing. When 



Figure 1. Measured cospectra in homogeneously sheared and salt-stratified turbulence, Schmidt number $\approx 700$. (a) Negative momentum flux and (b) density flux versus frequency $f$ in variance-preserving form at various downstream positions for $R i \approx 0$ and $R i \geq R i_{\mathrm{cr}} \approx 0.25$. This is a reproduction of Figures 20 and 22 from Rohr et al. (1988).
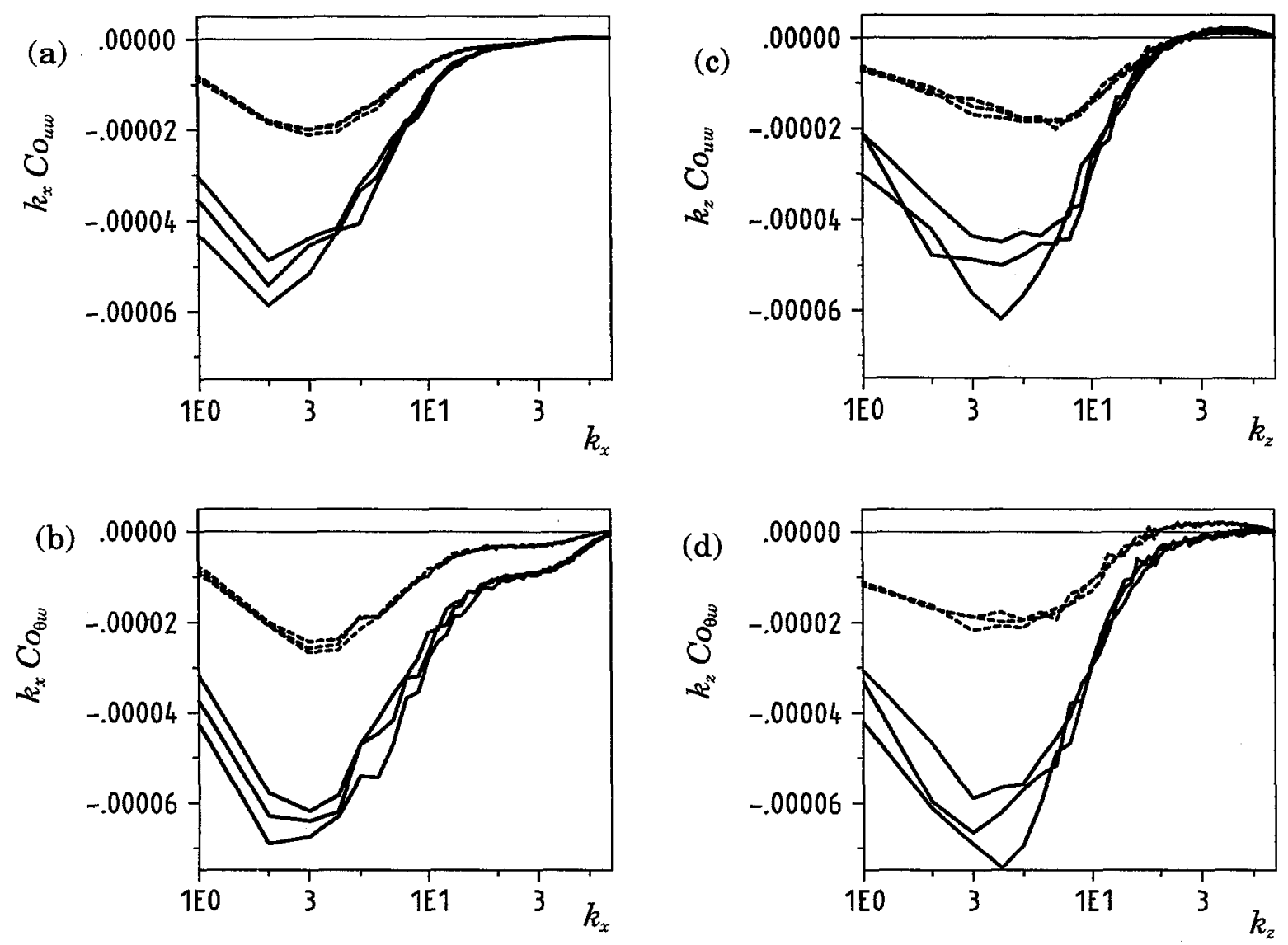

Figure 2. Simulated cospectra in homogeneously sheared and temperature-stratified turbulence with $\operatorname{Pr}=1$ at shear time $S t=6,7$, and 8. Data obtained from Kaltenbach et al. (1994) and related simulations. Downstream and vertical cospectra of momentum flux (a), (c) and heat flux (b), (d) in variance-preserving form for $R i=0$ (solid lines) and 0.13 (dashed lines). 




Figure 3. As Figure 2 for $R i=0.5$ (solid lines) and 1 (dashed lines) at $S t=8,9$, and 10 .

stratification is strong $\left(R i>R i_{\mathrm{cr}}\right), \mathrm{CG}$ fluxes occur at large scales which indicate collapsing turbulence. $R i_{\mathrm{cr}}$ is the critical Richardson number that separates flow with growing turbulent energy from those with decaying energy; its value lies between 0.13 and 0.25 (Schumann and Gerz, 1995). For our purposes the flux behavior at small scales is remarkable. There, we observe in both datasets that the momentum flux is CG for all $R i$ and the heat flux is not only zero for $R i=0$ but also CG when $R i>0$. At times $S t \geq 6$, the simulated flows have reached quasi-stationarity (Kaltenbach et al., 1994). Thus, the cospectra are representative for developed stages and their variation displayed for three different instants of time may be used as confidence intervals. The measured and the simulated data reveal that the observed CG fluxes, both at large and small scales, are persistent. In the following the small-scale flux behavior at different Richardson numbers is described and explained in detail.

\section{Explanation}

The idea that the small-scale PCG heat flux is driven by an excess of available potential energy at small scales, which can be caused either by weak dissipation of potential energy (when the Prandtl or Schmidt numbers are of order one or larger) or strong transfer of available potential energy from small to large wave numbers, i.e., large to small scales, stems from energy-budget considerations. Mechanistically, this effect can be understood in terms of warm rising and cold sinking fluid elements. Detailed descriptions of this process can, for example, be found in Schumann (1987), Holloway (1988), Gerz et al. (1989), and Gerz and Schumann (1991). We will see in Section 3 that the mechanistic model introduced below is also energetically consistent for the small-scale PCG momentum flux.

Here, we introduce a conceptional model of colliding fluid parcels that is able to explain PCG fluxes of momentum and heat at small scales (Sections 2.1-2.3). The model works for homogeneous turbulence 
under the influence of stable stratification with or without uniform mean shear. (A possible extension of this model to inhomogeneous boundary-layer flows is given in Section 3). The CG fluxes at large scales can be explained by the long-lasting influence of past mixing events, as discussed in Section 2.4. Further, the influence of the Prandtl number on PCG fluxes is described in Section 2.5.

It is well known that coherent structures of fluctuating vorticity develop from stretching and rotating interactions of the mean shear with the turbulent vorticity and velocity fields. In boundary-layer flows and in free shear flows, the dominant vortex structures resemble hairpins or horseshoes that are vertically inclined against the downstream direction by an angle of typically $25^{\circ}-35^{\circ}$ (Rogers and Moin, 1987; Gerz, 1991; Robinson, 1991). Far from boundaries, the horseshoe eddies can be oriented both upward and downward. We refer to them as "head-up" and head-down" structures. In a homogeneous shear flow with linear mean-velocity profile $U(z)$, both eddy structures occur equally often due to symmetric forcing. Gerz et al. (1994) conditionally sampled the vorticity fields in homogeneous shear flows, and found that the prominent feature is not a single eddy structure but a pair of eddies, where the head-up structure always lies below the head-down structure (Figure 4). A thermal microfront, i.e., a region with a large temperature gradient, and an enhanced shear layer, form in the zone between the eddies.

Figure 4 sketches a situation which is typical for weakly stably stratified and homogeneously sheared turbulence. Gerz et al. (1994) studied a flow with a Richardson number of $R i=0.13$. At such Richardson numbers, the flow is controlled by the shear and stratification does not significantly suppress or fundamentally alter the turbulence, compared with a neutrally stratified condition. Hence, we can assume that the situation sketched in Figure 4 is also typical for neutrally stratified shear flows, where temperature represents a passive scalar field.

On the other hand, flows with large values of the Richardson number are characterized by the weak influence of shear and the strong influence of stratification such that the turbulence decays with time. Such flows do not exhibit horseshoe-vortex structures at quasi-stationary states. Vorticity, rather, is organized in horizontal sheets and streaks (Gerz, 1991).

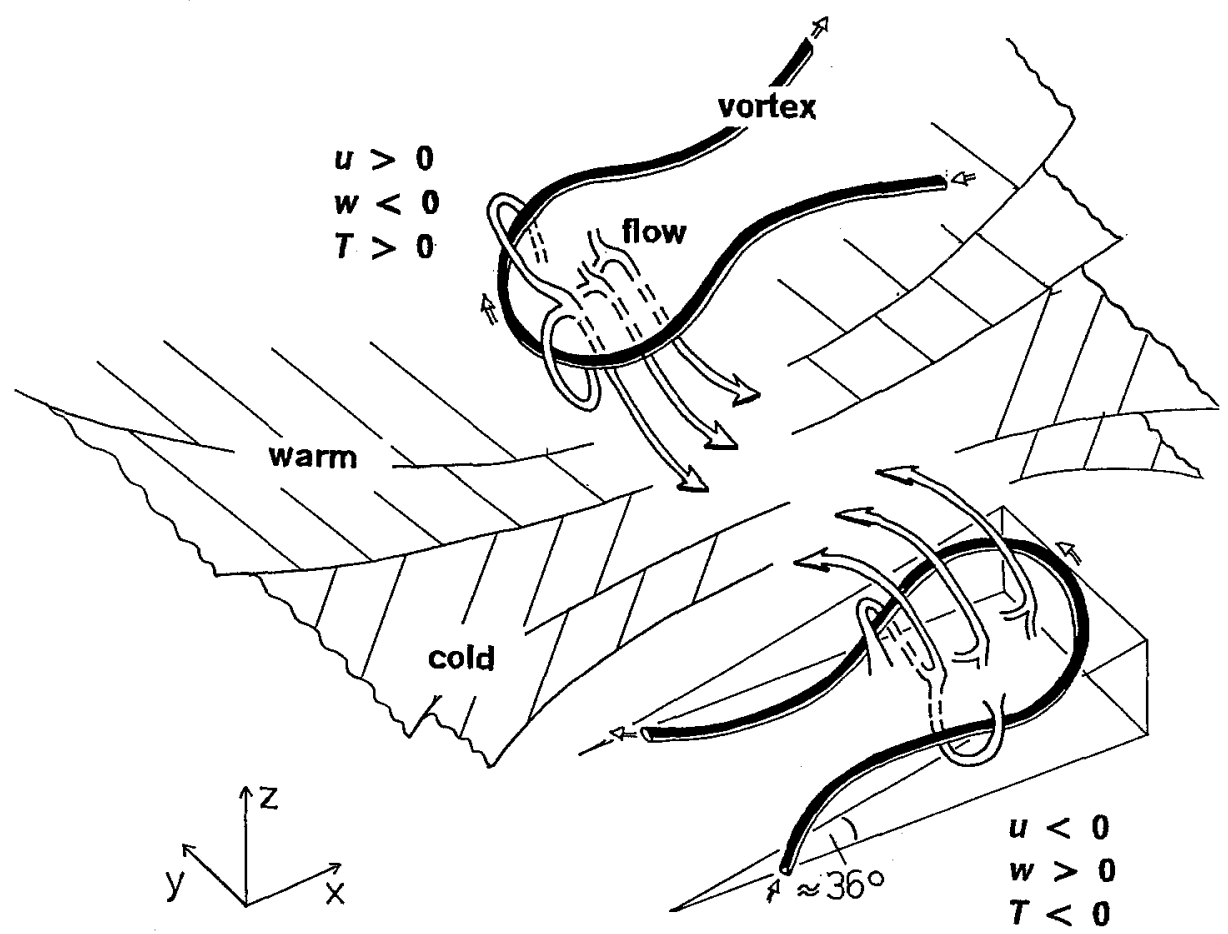

Figure 4. Sketch of a pair of vortex structures as it typically occurs in homogeneously sheared turbulence at neutral and weakly stable stratification with $d U / d z>0$. A head-down eddy lies above a head-up eddy and a convergence zone develops between them. (From Gerz et al., 1994). 


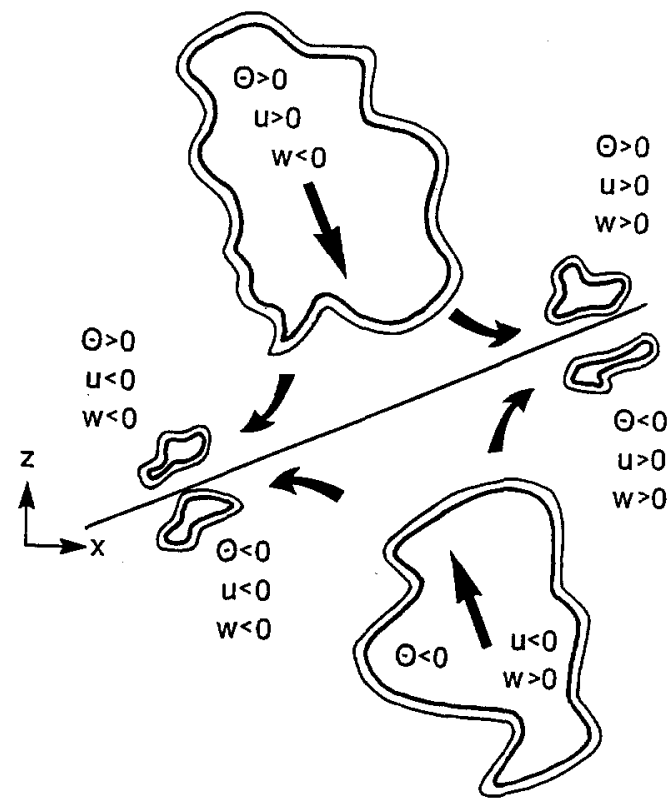

Figure 5. Two-dimensional sketch of the convergence zone where collision takes place in the $x-z$ plane. The large fluid lumps are advected through the legs of their parent eddy structures and collide. Small fluid parcels result in the four sectors with properties as labeled. The sketch illustrates a typical situation for unstratified shear flow, $R i=0, d U / d z>0$. The straight line symbolizes the microfront.

\subsection{Case I: Neutrally Stratified Shear Flow}

First, we consider neutrally stratified shear flows, $R i=0$, where temperature is passive. In this flow the mean shear $S$ drives the turbulence to become more and more energetic. In Figures 1 and 2 we recognize dominant DG fluxes of momentum and heat (density) at wave numbers $k_{x}$ (or frequency $f$ ) and $k_{z}$ in the range $1 \leq k<10$, hence, at large scales. However, at small scales $(k>25)$, the momentum flux is CG, whereas the density (heat) flux is weakly DG or zero. We note that the density flux at $R i=0.01$ to 0.03 in Rohr's experiment (Figure 1(b)) can be considered as a passive scalar case. No positive fluxes are found in cross-stream $\left(k_{y}\right)$ spectra of the numerically simulated turbulence.

In homogeneous turbulence the horseshoe vortices are responsible for the strong DG fluxes at large and medium scales (Gerz, 1991). Between their legs, the vortices transport very efficiently heavy (cold) and slow fluid lumps upward (head-up eddy) or light (warm) and fast fluid lumps downward (head-down eddy), see Figure 4. Since the structures happen to occur mostly as pairs in homogeneous turbulence (see Gerz et al., 1994), they transport the lumps toward each other, with the result that the lumps collide in the frontal zone between the parent eddy structures and break into smaller parcels. During collision, fluid lumps exchange momentum by pressure forces but exchange heat only when they mix. This picture of colliding fluid lumps provides an interpretation of the mixing process in terms of dynamic actions of flow structures. The small-scale CG momentum flux and the vanishing heat flux at small scales result from this collision: as sketched in Figure 5, four different sectors have to be distinguished schematically in the collision area, above and below the microfront and left and right of the collision center. (Since the mean flow is symmetric with respect to the cross-stream direction $y$, it is sufficient to consider the collision zone in a downstream/vertical cross section only.) For $S>0$, every sector provides a positive $u w$. During collision we can assume that the four smaller parcels resulting from collision maintain most of their primary heat such that we observe a positive and a negative $\theta w$ on each side of the front. Hence, in the mean $(\langle\rangle$, averaged over the four sectors), net fluxes $\langle u w\rangle>0$ and $\langle\theta w\rangle=0$ result consequently. ${ }^{1}$

\footnotetext{
${ }^{1}$ In the averaged cospectra from the simulations, we observe a stronger CG effect in the vertical spectra than in the downstream spectra. This reflects the fact that the vortex structures are vertically inclined with an angle smaller than $45^{\circ}$. As a consequence, the spectral character of these structures dominate more in averaged $k_{x}$ spectra than in averaged $k_{z}$ spectra. Hence, they hide the spectral character of the collided fluid parcels almost completely in downstream spectra but only marginally in vertical spectra. A spectral evaluation of the data in tilted directions parallel and perpendicular to the horseshoe eddies revealed this effect at its strongest extent.
} 


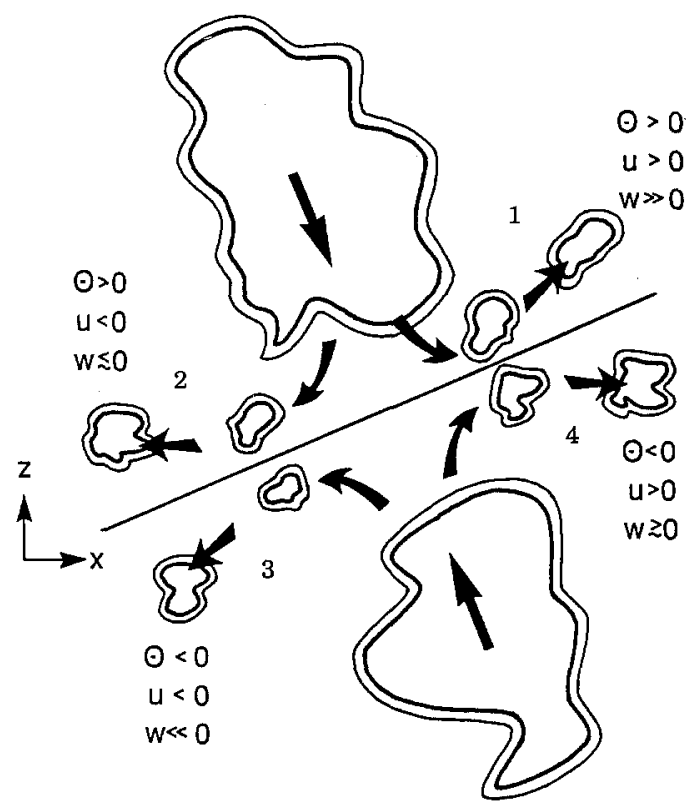

Figure 6. As Figure 5. The resulting flow properties in the four sector are typical for a stably stratified but shear-controlled flow, $R i=0.13$.

\subsection{Case II: Weakly Stratified Shear Flow}

Here, we look at weakly stratified shear flows, $R i=0.13$. Temperature is now an actively buoyant scalar that reduces turbulent mixing. Therefore, the fluxes at large scales are weaker than in the passive case, see Figure 2. Further, the simulation data show that turbulence has approximately reached a stationary state (Kaltenbach et al., 1994). Qualitatively, the cospectra of $u w$ do not differ from those in case I. However, we observe a reduced DG heat flux at large $k_{x}$ and a positive (CG) heat flux at large $k_{z}$ (Figure 2(b), (d)).

Again, the warm and cold fluid lumps which are advected through the legs of the parent eddy structures collide in the frontal zone and break into smaller parcels essentially maintaining their primary heat. However, now these parcels are actively warm and cold, i.e., a warm parcel will rise or resist the dynamically forced sinking due to its buoyancy; a cold parcel will sink or resist the dynamically forced rising, as illustrated for four typical parcels in Figure 6. There, parcel 1 (3) will enhance the dynamically forced rising (sinking), whereas parcel 2 (4) will only diminish the dynamically forced sinking (rising). In other words, the former mechanism strengthens the positive heat fluxes in parcels 1 and 3, but the latter mechanism weakens the negative heat fluxes in parcels 2 and 4 . This asymmetric behavior of the parcels results in a positive net heat flux at small scales. The momentum flux is explained as in case I.

\subsection{Case III: Strongly Stratified Shear Flow}

In the last case we study strongly stratified shear flows, $R i=0.5$ and 1 . These flows resemble situations of decaying turbulence. For both Richardson numbers, the covariance spectra of momentum flux and heat flux in Figure 3 show positive contributions at large and at small $k_{x}$, whereas they are negative at medium wave numbers. At small vertical wave numbers the fluxes show negative or indifferent values, but at medium and large $k_{z}$ the fluxes are strongly CG. Positive fluxes at large scales are also found in $k_{y}$ spectra of the simulations. The cospectra at large Ri measured by Rohr et al. (1988) are depicted only as an envelop over all values in Figure 1. However, the data corroborate our observations from the simulations qualitatively.

In contrast to shear controlled flows, horseshoe eddies do not exist in flows with zero mean shear or strong stratification (Gerz, 1991), but warm and cold fluid lumps buoyantly rise or sink and collide occasionally (see, e.g., Gerz et al., 1989; Gerz and Yamazaki, 1993). These colliding lumps are of medium size, $10<k<30$, and break into smaller parcels $(k>30)$. Figure 7 sketches this situation for weak shear. Before collision, the lumps are buoyancy-driven and carry a CG heat flux and a DG or zero momentum flux 


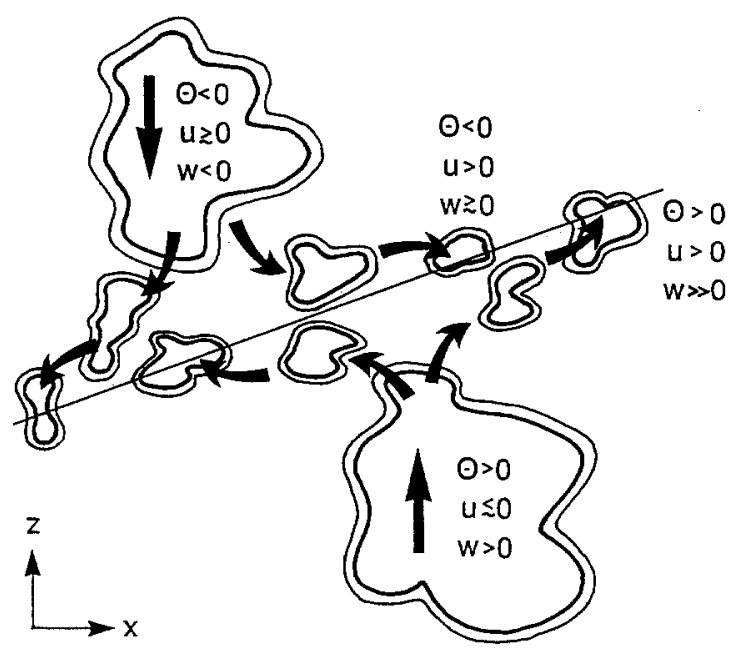

Figure 7. Two-dimensional sketch of a collision of fluid lumps which follow their own buoyancy force. The resulting flow properties in the four sectors are typical for strong stratification and weak shear, $R i=0.5$ and 1 .



Figure 8. Measured downstream cospectra of the vertical turbulent heat flux in homogeneous temperature-stratified flow without shear at two downstream positions. The flow had a mesh Froude number of 5 and $\operatorname{Pr} \approx 5$. This is a reproduction of a figure by Komori and Nagata (1994).

at wave numbers roughly between 10 and 30 . The cospectra in Figure 3 corroborate this scenario, especially for $R i=0.5$ and as a function of $k_{z}$ : The strong CG contribution of $\theta w$ in the range of $10<k<30$ is due to the buoyantly moving medium-sized lumps. The observed CG fluxes at the small scales $(k>30)$ result from the situation after collision and are explained as in case II.

For positive shear, $S>0$, the collision front between two buoyantly moving lumps is on average inclined against the mean flow direction (the positive angle between the $x$-direction and the front). The inclination of the collision front is the reason why the net momentum flux at small scales is CG. In the absence of a mean shear, the lumps collide under any angle, such that the net small-scale momentum flux, averaged over all collisions in the domain, is zero. Hence, the collision model is also consistent with the observations made in shear-free stratified flows, where the mean momentum flux is indeed zero, but the small-scale heat flux is persistently CG. Figure 8 shows downstream cospectra of the vertical heat flux of grid-generated turbulence in an unsheared but stratified water tank with $P r=5$ at two downstream positions. These spectra have recently been measured by Komori and Nagata (1994). We recognize that the heat flux oscillates at large scales but remains persistently CG at small scales. This result is in perfect agreement with the predictions of the collision model. Previous measurements of unsheared and stratified turbulence in air flows with $P r=0.7$ (Lienhard and van Atta, 1990; Yoon and Warhaft, 1990) show very small fluxes at small scales and no significant $C G$ fluxes. The different results could be caused by the different Prandtl numbers of the fluids (the influence of $\mathrm{Pr}$ is discussed in Section 2.5).

\subsection{Persistent CG Fluxes at Large Scales}

The CG fluxes at large scales depend on the kind of forcing and, therefore, may look different in the atmosphere, the ocean, or the laboratory. In our case the PCG fluxes of momentum and heat at the largest scales, as displayed for $k<10$ in Figure 3, occur only at strong stratification $\left(R i>R i_{\mathrm{cr}}\right)$, when the flow is controlled by buoyancy and decays in time. The fluxes can be explained by the long-lasting influence of past mixing events. Kaltenbach et al. (1994) mentioned this effect previously, but did not provide a detailed explanation. To our understanding the large-scale CG momentum flux develops as follows: if there was 
a strong negative correlation between $u$ and $w$ in a sheared and stratified flow at early times, and if the fluid lump has not yet adjusted its horizontal velocity to the mean velocity of its new surroundings when $w$ changes sign for restratification, then $\langle u w\rangle$ will become positive (where \langle\rangle denotes an appropriate mean). ${ }^{2}$ The same process causes a PCG heat flux at large scales, since $u$ and $\theta$ are strongly correlated (Rohr et al., 1988; Holt et al., 1992; Kaltenbach et al., 1994). The largest scales are affected by buoyancy first, causing the sign change of $w$. Indeed, Figure 3(a), (b) shows that this type of CG flux starts at the largest wave numbers and later affects smaller and smaller scales when $R i$ is increased.

Flow history at large scales becomes important for the evolution of turbulence when the transfer of energy from large scales in the production range to small scales in the dissipation range is reduced. In other words, motions at small scales are decoupled from those at large scales when the turn-over timescale increases relative to the dissipative timescale. As shown in Figure 11 later, this is observed for flows with large Richardson numbers. Hence, strongly stratified flows suffer more from flow history (e.g., initial conditions) than flows with weak stratification.

\subsection{Influence of the Prandtl Number}

The molecular (or, in the case of LES, the subgrid-scale) Prandtl number Pr affects the dissipation rate of available potential energy (or temperature variance) but not the dissipation rate of kinetic energy (velocity variances). Therefore, a change of $\operatorname{Pr}$ can affect the small scales of the heat flux directly but the momentum flux only indirectly and to a weaker extent. This is corroborated by the graphs in Figure 9

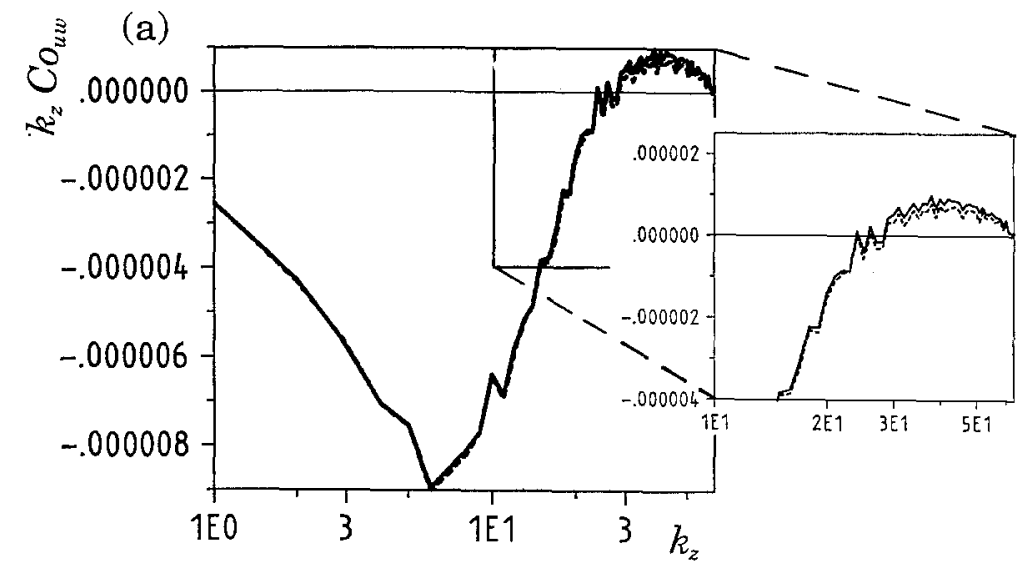

(b)

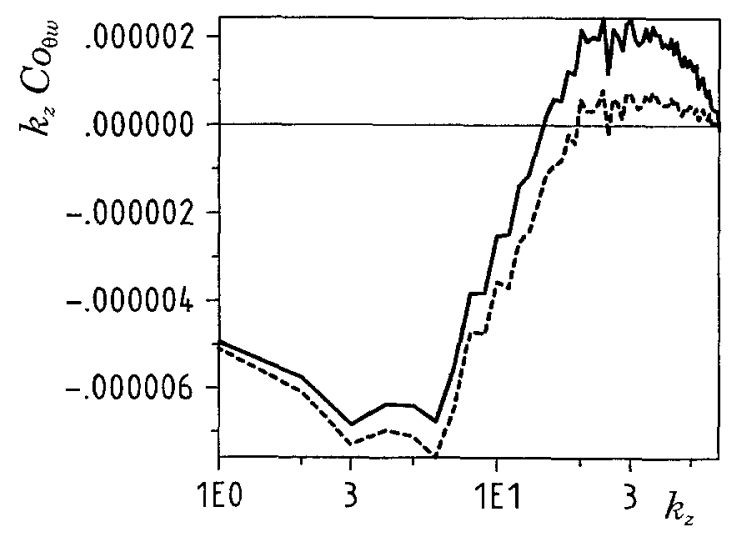

Figure 9. Vertical cospectra of momentum flux (a) and heat flux (b) in variance-preserving form for $R i=0.25$ at $S t=7$ (from Kaltenbach et al., 1994). Solid line: $P r=1$, dashed line: $P r=0.5$.

\footnotetext{
${ }^{2}$ Kaltenbach et al. (1991) thought to explain the small-scale CG fluxes by this mechanism.
} 
depicting the flux cospectra for $R i=0.25$ for $P r=1$ and 0.5 (LES data). We now show that the conclusions drawn from the collision model for flows with $\operatorname{Pr} \geq 1$ are also consistent with the CG-flux behavior in flows with $P r=0.5$.

If $\operatorname{Pr}$ is 1 or larger, temperature fluctuations are diffused at a weaker rate than velocity fluctuations (Gerz et al., 1989; Gerz and Schumann, 1991). Thus, the parcels keep their temperature properties longer than their velocity properties, and, consequently, warm and cold, slowly rising and sinking spots develop: Buoyancy enhances the vertical motion of parcels 1 and 3 and changes the sign of the vertical velocity of parcels 2 and 4 (Figure 6). The related momentum fluxes of parcels 2 and 4 then become DG again. Hence, we expect scales where the heat flux is CG but the momentum flux is DG. This is corroborated in Figure 2(c), (d) for $R i=0.13$ (see range $15<k<30$ ) and the trend is enhanced for larger $R i$ (see Figure 3 and Rohr's data in Figure 1).

On the other hand, when $\operatorname{Pr}$ is smaller than about $1^{3}$ - as for the wind-tunnel experiments by Lienhard and van Atta (1990) and Yoon and Warhaft (1990) - the rate of dissipation of temperature variance increases relative to the dissipation rate of velocity variance. Thus, the small fragments are less buoyant and mostly driven by the collision dynamics (see Figure 6). So, parcels 1 and 3 produce a CG heat flux, whereas parcels 2 and 4 sustain a weakly DG heat flux. The CG momentum flux is observed at all four parcels. Hence, the average CG heat flux is strongly reduced, whereas the average CG momentum flux is only slightly (but systematically) diminished compared with cases with larger $\operatorname{Pr}$ values (dashed lines in Figure 9). The fact that the small-scale momentum flux for $R i>0$ is sensitive to the value of the Prandtl number (see close-up in Figure 9(a)) results from buoyancy that increases the vertical velocity of parcels after collision when $\operatorname{Pr}>1$.

\section{Discussion}

In shear-controlled homogeneous turbulence, the inclined eddies mostly occur in pairs (Gerz et al., 1994), such that the advected fluid lumps form a microfrontal zone between the structures (Figure 4). From Figures 5 and 6, we learn that the net properties of the small-scale parcels are provided by the average over the parcels on each side of the microfront. Hence, a pair of eddy structures is not necessary to explain the development of the PCG fluxes. The same arguments also hold for a single eddy structure where the fluid lump between the legs of the parent eddy collides with noncoherently moving fluid masses. For example, in a boundary-layer flow close to the lower boundary, only head-up structures can exist. We expect that CG fluxes at small scales are also present in such cases. Härtel and Kleiser(1993) indeed report such fluxes in the buffer layer of their channel-flow simulations and suggest that such reversed flow is associated with the ejection events. It is possible that ejections transporting fluid with small downstream momentum upward $(u w<0)$ are caused by head-up vortices. Hence, the observed CG momentum flux is probably produced when the ejected fluid collides with downward-moving fluid of higher downstream momentum.

The findings from the collision model are also consistent with the observed cospectra of the horizontal heat flux, $\theta u$, shown in Figure 10. The model predicts an average nonzero horizontal heat flux despite the absence of a respective mean gradient (note that $d \Theta / d x=0$ ). The predictions are also consistent with the behavior of $\theta u$ at different scales: In case I we expect large positive values of $\theta u$ at large scales and vanishing values at small scales (see Figure 5). When the flow is stably stratified, as in cases II and III, $\theta u$ should decrease at large scales, since stable stratification reduces mixing. However, at small scales, the horizontal heat flux should slightly increase relative to the large scales, because parcels 1 and 3 in Figure 6 are more energetic than parcels 2 and 4 . This means that $u$ of parcels 1 and 3 increases in magnitude at the expense of $w$ by the action of pressure, whereas $w$ of parcels 2 and 4 is too small to feed $u$. Therefore, we expect an average positive heat flux at small scales, $\langle\theta u\rangle>0$. The plots in Figure 10 corroborate our model since, with increasing stratification, the heat flux at small scales gains more and more intensity relative to the decaying mean value.

In flows with molecular Prandtl numbers of order 1 or less, such as air, the CG fluxes occurring at small scales have a minor effect on the entire flow evolution. However, in flow with large Prandtl or Schmidt numbers such as water or a salt-water solution, the small parcels that result from colliding larger fluid lumps keep their temperature properties longer than their velocity properties because of different dissipation rates

\footnotetext{
${ }^{3}$ The limit between small and large values of $\operatorname{Pr}$ should be the effective turbulence Prandtl number of neutral turbulence. This limit is a little less than 1 (Schumann and Gerz, 1995).
} 

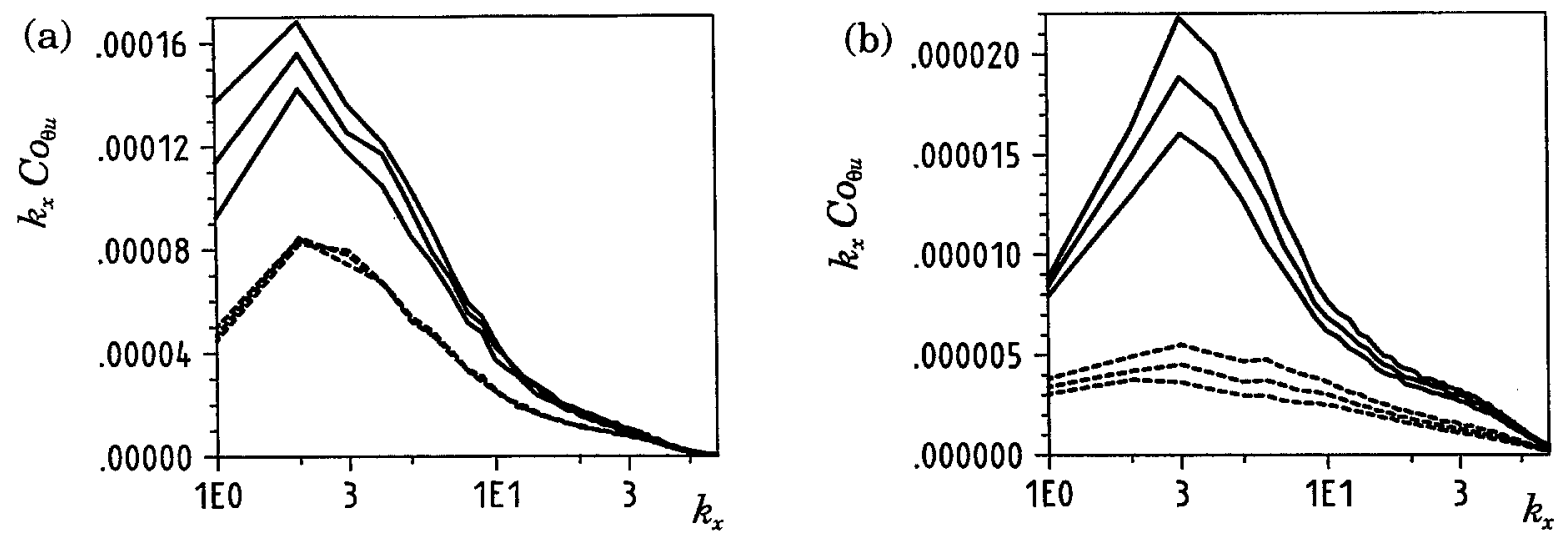

Figure 10. Simulated downstream cospectra of the horizontal heat flux; (a) $R i=0$ (solid lines) and 0.13 (dashed lines) at $S t=6,7$, and 8; (b) $R i=0.5$ (solid lines) and 1 (dashed lines) at $S t=8,9$, and 10. (LES data, see Kaltenbach et al., 1994.)

for available potential and kinetic energy (Schumann, 1987). Hence, these small parcels may become buoyancy-driven. The resulting CG heat flux is more intense than in flows with $\operatorname{Pr}$ of order 1, as measured by Komori and Nagata (1994) (see also Figure 8) and simulated by Gerz and Yamazaki (1993). Such parcels are embedded in the (wavy) large-scale flow as warm and cold, slowly rising the sinking spots, and may become a dominant flow property at strong stratification. Such a situation is, e.g., typical for the oceanic thermocline with a Schmidt number of about 8 . In the thermocline, patches of enhanced temperature fluctuations are often observed without corresponding signals in the fluctuating velocity field (see Munk, 1981). Our collision model provides a tool to explain these patches dynamically, i.e., as a result of collsions of large-scale fluid lumps driven either by shear or by buoyancy.

The phenomenon of CG momentum flux is subject of a book by Starr (1968). He mostly describes transient and persistent flux phenomena at large scales (e.g., between wave numbers 1 and 15 on the planetary scale of the earth's atmosphere, (Starr, 1968, p. 48)). However, he formulates an energetic condition for the appearance of such fluxes which is also relevant for our discussion of CG fluxes at small scales: the CG motion must be supplied by kinetic energy at the respective scales, either by conversion from other energy reservoirs or by transfer of kinetic energy from other scales to the CG scales (Starr, 1968, p. 23). Both situations are given at the small scales of the flows considered here:

(i) A CG motion gains energy by conversion of available potential energy to kinetic energy when the flow with a large Prandtl number is stably stratified, as discussed in detail above.

(ii) In addition to this source, we suppose that the CG momentum flux at small scales results from a quick transfer of energy from the production range to small scales by the collision process causing accumulation of energy in the dissipation range.

Evidence for the quick spectral transfer of energy may be seen in the different evolutions of the timescales for turn-over $\left(l / E^{1 / 2}\right)$ and dissipation $(E / \varepsilon)$, where $E$, $\varepsilon$, and $l$ stand for turbulent kinetic energy, its dissipation rate, and the integral length, respectively. By plotting the ration $E^{3 / 2} /(\varepsilon l)$ versus shear time $S t$ in Figure 11, we see that the ratio is larger than 1 and that it even grows in time after $S t \approx 2$ for the most energetic cases $(R i=0$ and 0.13$)$. Thus, when the flow evolves, the energy cascades quicker from large to small eddies than it is removed by viscosity in the dissipation range. To counteract energy accumulation, the flow produces CG motion (with CG momentum fluxes) at the small scales.

On the other hand, for the cases with $R i \geq 0.5$, the timescale ration decreases with $S t$. This reflects the growing suppression of the energy cascade causing separation of the motion at small scales from the motion at large scales and indicates a growing incluence of flow history as discussed in Section 2.4. Now, the small scales gain energy from the buoyantly moving medium-sized lumps when they occasionally collide and break and, as explained above, from conversion of available potential energy to kinetic energy if the Prandtl number is larger than 1 . Hence, molecular diffusivities are more important for stratified flows than for nonstratified flow because buoyancy reduces the energy-cascading process and diminishes coupling between the energy levels at small and large scales. 


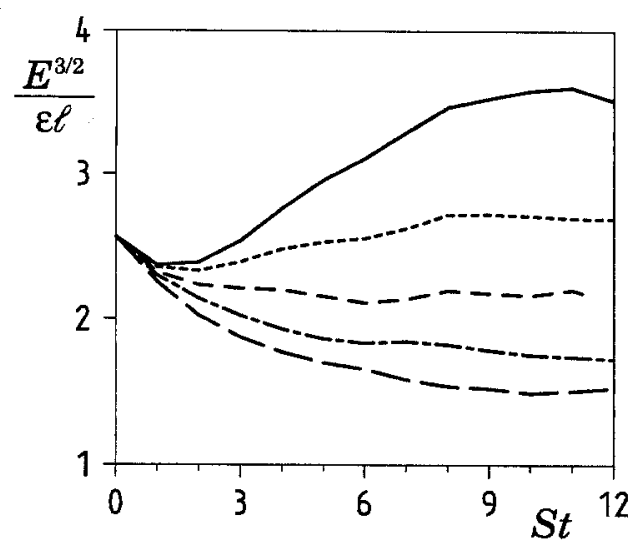

Figure 11. Ratio of dissipation timescale, $E / \varepsilon$, and turn-over timescale, $l / E^{1 / 2}$, versus $S t ; R i=0:-; R i=0.13:-.---; R i=0.25:-\_-; R i=0.5$ : $-\ldots$ -; Ri $=1$ : ——. (LES data, see Kaltenbach et al., 1994.)

\section{Summary and Conclusion}

We addressed the phenomenon of PCG vertical fluxes of downstream momentum and heat in homogeneously sheared and stratified turbulence. We defined a PCG flux as a flux that persists for longer than half the buoyancy period $\pi / N$ of the stratified fluid. PCG fluxes are observed in many flow situations, but they seem to contradict general (thermodynamical) expectations. We showed that one has to distinguish between PCG-flux phenomena at large and at small scales, and that they require different explanations. We presented a conceptional model to explain the development of PCG fluxes of momentum and heat at small scales.

We summarize our findings as follows:

- PCG fluxes of momentum and heat are not restricted to low Reynolds and high Prandtl number flows, because they have been documented in various measured and simulated flow situations with small to at least moderate Reynolds numbers (up to 200 based on microscales) and with Prandtl or Schmidt numbers varying from 0.5 to several hundreds (see, e.g., Komori et al., 1983; Itsweire et al., 1986; Rohr et al., 1988; Gerz et al., 1989; Gerz and Schumann, 1991; Holt et al., 1992; Ramsden and Holloway, 1992; Komori and Nagata, 1994; Kaltenbach et al., 1994).

- As mentioned previously by Kaltenbach et al. (1994), the PCG fluxes at large scales evolve under strong stratification, i.e., at large Richardson numbers, only. They are controlled by flow history, that is, the fluxes result from effects which are caused by past events with a long-lasting influence on the flow evolution, such as a strong disequilibrium between kinetic and available potential energy of the flow. Hence, initial and boundary conditions become important in this case.

- Conversely, the PCG fluxes at small scales occur at all positive (for momentum even at zero) Richardson numbers. The basic physical process is the break-up of energetic large-scale fluid lumps into small-scale parcels in the collision zone: In a shear-dominated flow the collision is driven by inclined coherent vorticity structures, the horseshoe eddies; in flows controlled by stratification (with weak or zero shear), large-scale lumps move in the gravity field driven by their own buoyancy and the most energetic ones occasionally collide. The inclination due to the mean shear is the mechanistic reason why the net momentum flux at small scales is also CG. In the absence of a mean shear, the lumps collide under any angle, such that the net small-scale momentum flux averaged over all collisions is zero. Hence, the model applies to flows with and without mean shear.

- The model of the PCG-flux dynamics may also explain why ejection events in boundary-layer flows cause (weak) CG momentum fluxes as reported by Härtel and Kleiser (1993).

- The collision model predicts the correct flux behavior at small scales as measured and simulated for a variety of Richardson numbers ranging from 0 to 1 . It exhibits the right trend in the small-scale heat flux when the Prandtl number is reduced from values larger than 1 to 0.5 , and it explains why the small-scale momentum flux is also weakly but systematically influenced by $\mathrm{Pr}$.

- The collision model is consistent with energy conditions as expressed by $\operatorname{Starr}(1968$, p. 23). The kinetic energy of the small-scale CG motions is supplied by the transfer of energy from larger (productionrange) scales to the CG scales and by conversion of available potential energy into kinetic energy at the CG scales when the flow is stably stratified and $\mathrm{Pr}>1$. 
Finally, we conclude that the small-scale PCG fluxes of momentum and heat are a universal property of stably stratified homogeneously sheared turbulence. On the other hand, the PCG fluxes at large scales depend on the influence of flow history and, hence, are not universal.

\section{Acknowledgments}

The authors wish to thank Prof. Komori, Mr. Nagata, Prof. van Atta, and Dr. Rohr for placing their original figures at our disposal and Dr. Kaltenbach for a series of discussions. The comments and hints of the anonymous referees are gratefully acknowledged.

\section{References}

Gerz, R. (1991). Coherent structures in stratified turbulent shear flows deduced from direct simulations. In: Turbulence and Coherent Structures (eds. O. Métais and M. Lesieur), Kluwer, Dordrecht, pp. 449-468.

Gerz, T., and Schumann, U. (1991). Direct simulations of homogeneous turbulence and gravity waves in sheared and unsheared stratified flows. In: Turbulent Shear Flow, vol. 7 (eds. F. Durst et al.), Springer-Verlag, New York, pp. $27-45$.

Gerz, T., and Yamazaki, H.(1993). Direct numerical simulation of buoyancy driven turbulence in stably stratified fluid. J. Fluid Mech., 249, 415-440.

Gerz, T., Schumann, U, and Elghobashi, S.E. (1989). Direct numerical simulation of stratified homogeneous turbulent shear flows. J. Fluid Mech., 200, 563-594.

Gerz, T., Howell, J., and Mahrt, L. (1994). Vortex structures and microfronts. Phys. Fluids A, 6, 1242-1251.

Härtel, C., and Kleiser, L. (1993). Large-eddy simulation of transitional and turbulent wall-bounded flows. In: Flow Simulation with High-Performance Computers, vol. I (ed. E.H. Hirschel), Vieweg-Verlag, Braunschweig, pp. 169-185.

Holloway, G. (1988). The buoyancy flux from internal gravity wave breaking. Dyn. Atmos. Oceans, 12, 107-125.

Holt, S.E., Koseff, J.R., and Ferziger, J.H. (1992). A numerical study of the evolution and structure of homogeneous stably stratified sheared turbulence. J. Fluid Mech., 237, 499-539.

Itsweire, E.C., and Helland, K.N. (1989). Spectra and energy transfer in stably stratified turbulence. J. Fluid Mech., 207, 419-452.

Itsweire, E.C., Helland, K.N., and van Atta, C.W. (1986). The evolution of grid-generated turbulence in a stably stratified fluid. $J$. Fluid Mech., 162, 299-338.

Kaltenbach, H.-J., Gerz, T., and Schumann, U. (1991). Transport of passive scalars in neutrally and stably stratified homogeneous turbulent shear flows. In: Advances in Turbulence, vol. 3 (eds. A. Johansson and P. Alfredsson). Springer-Verlag, Berlin, pp. 327-334.

Kaltenbach, H.-J., Gerz, T., and Schumann, U. (1994). Large-eddy simulation of homogeneous turbulence and diffusion in stably stratified shear flow. J. Fluid Mech., 280, 1-40.

Komori, S., and Nagata, K. (1994). Heat and mass transfer in stably stratified flows. Presented at Fourth Internat. Symp. on Stratified Flows, June 29 to July 2, 1994, Grenoble.

Komori, S., Ueda, H., Ogino, F., and Mizushina, T. (1983). Turbulence structure in stably stratified open-channel flow. J.Fluid Mech., 130, $13-26$.

Lienhard, J.H., and van Atta, C.W. (1990). The decay of turbulence in thermally stratified flow. J. Fluid Mech., 210, 57-112.

Munk, W.H. (1981). Internal waves and small-scale processes. In: Evolution of Physical Oceanography (eds. B.A. Warren and C. Wunsch). MIT Press, Cambridge, MA, pp. 264-291.

Ramsden, D., and Holloway, G. (1992). Energy transfers across an internal wave-vortical mode spectrum. J. Geophys. Res., 97, 3659-3668.

Robinson, S.K. (1991). Coherent motions in the turbulent boundary layer. Annu. Rev. Fluid Mech., 23, 601-639.

Rogers, M.M., and Moin, P. (1987). The structure of the vorticity field in homogeneous turbulent flows. J. Fluid Mech., 176, 33-66.

Rohr, J.J., Itsweire, E.C., Helland, K.N., and van Atta, C.W. (1988). Growth and decay of turbulence in a stably stratified shear flow. J. Fluid Mech., 195, 77-111.

Salathé, E.P., and Smith, R.B. (1992). In situ observations of temperature microstructure above and below the tropopause. J. Atmos. Sci., 49, 2032-2036.

Schumann, U. (1987). The countergradient heat-flux in turbulent stratified flows. Nuclear. Engrg. Des., 100, $255-262$.

Schumann, U., and Gerz, T. (1995). Turbulent mixing in stably stratified shear flows. J. Appl. Meteorol., 34, 33-48.

Starr, V.P. (1968). Physics of Negative Viscosity Phenomena. McGraw-Hill, New York, 256 pp.

Yoon, K. and Warhaft, Z. (1990). The evolution of grid generated turbulence under conditions of stable thermal stratification. J. Fluid Mech., 215, 601-638. 